\title{
A UNIQUENESS RESULT FOR THE FOURIER TRANSFORM OF MEASURES ON THE SPHERE
}

\author{
FRANCISCO JAVIER GONZÁLEZ VIELI
}

\author{
(Received 16 August 2011)
}

\begin{abstract}
A finite measure supported by the unit sphere $\mathbb{S}^{n-1}$ in $\mathbb{R}^{n}$ and absolutely continuous with respect to the natural measure on $\mathbb{S}^{n-1}$ is entirely determined by the restriction of its Fourier transform to a sphere of radius $r$ if and only $2 \pi r$ is not a zero of any Bessel function $J_{d+(n-2) / 2}$ with $d$ a nonnegative integer.
\end{abstract}

2010 Mathematics subject classification: primary 42B10; secondary 46F12.

Keywords and phrases: Heisenberg uniqueness, Fourier transform, measure, sphere.

\section{Introduction}

Hedenmalm and Montes-Rodríguez asked in [4] the following: given $\Gamma$ a smooth curve in $\mathbb{R}^{2}$ and $\Lambda$ a subset of $\mathbb{R}^{2}$, when is it possible to recover uniquely a finite measure $v$ supported by $\Gamma$ and absolutely continuous with respect to the arc length measure on $\Gamma$ from the restriction to $\Lambda$ of its Fourier transform $\mathcal{F} v$ on $\mathbb{R}^{2}$ ? Equivalently, when does $\mathcal{F} v(\lambda)=0$ for all $\lambda \in \Lambda$ imply $v=0$ ? If this is the case, they call $(\Gamma, \Lambda)$ a Heisenberg uniqueness pair.

Then, among other results on Heisenberg uniqueness, Lev and Sjölin established independently that if $\Gamma$ is the unit circle $\mathbb{S}^{1}$ and $\Lambda$ is a circle of radius $r,(\Gamma, \Lambda)$ is a Heisenberg uniqueness pair if and only if $r$ is not a zero of any Bessel function $J_{n}$ $\left(n \in \mathbb{N}_{\geq 0}\right)$ (see [5, Theorem 1(i), p. 135] and [6, Theorem 1(i), p. 126]).

The definition of Heisenberg uniqueness pairs can easily be extended to all $\mathbb{R}^{n}$ $(n \geq 2)$ :

Definition 1.1. Let $\Sigma$ be a $C^{1}$ submanifold of $\mathbb{R}^{n}(n \geq 2), \mu_{\Sigma}$ the natural measure on $\Sigma$ and $\Lambda$ a subset of $\mathbb{R}^{n}$. The pair $(\Sigma, \Lambda)$ is a Heisenberg uniqueness pair if, for every finite measure $v$ on $\Sigma$ which is absolutely continuous with respect to $\mu_{\Sigma}, \mathcal{F} v(\lambda)=0$ for all $\lambda \in \Lambda$ implies $v=0$, where $\mathcal{F} v$ is the Fourier transform of $v$ on $\mathbb{R}^{n}$ :

$$
\mathcal{F} v(x)=\int_{\Sigma} e^{-2 \pi i x \cdot \eta} d v(\eta)
$$

for all $x \in \mathbb{R}^{n}$.

(C) 2011 Australian Mathematical Publishing Association Inc. 0004-9727/2011 \$16.00 
We have obtained the following criterion for the Heisenberg uniqueness of pairs of spheres.

Proposition 1.2. Let $\mathbb{S}^{n-1}$ be the sphere with centre 0 and radius 1 in $\mathbb{R}^{n}$ and $\Lambda$ a sphere of radius $r$. The pair $\left(\mathbb{S}^{n-1}, \Lambda\right)$ is a Heisenberg uniqueness pair if and only if $J_{d+(n-2) / 2}(2 \pi r) \neq 0$ for all $d \in \mathbb{N}_{\geq 0}$.

Since we must do without the group structure available on the unit circle, our proof is not as short as the one in dimension two. So, before giving it in Section 3, we recall in Section 2 some useful facts.

\section{Preliminaries}

If $(\Sigma, \Lambda)$ is a Heisenberg uniqueness pair in $\mathbb{R}^{n}$, it follows from elementary properties of the Fourier transform that $(\Sigma, \Lambda+b)$ is also a Heisenberg uniqueness pair for any $b \in \mathbb{R}^{n}$.

By the theorem of Radon-Nykodým, a measure $v$ is absolutely continuous with respect to a measure $\mu$ if and only if $v$ has a density function $f$ with respect to $\mu$, that is, $v=f \cdot \mu$. Moreover, if $v$ is finite, then $f$ is integrable with respect to $\mu$.

We write $d \sigma$ for the natural measure on $\mathbb{S}^{n-1}$. A spherical harmonic of degree $l$ on $\mathbb{S}^{n-1}\left(l \in \mathbb{N}_{\geq 0}\right)$ is the restriction to $\mathbb{S}^{n-1}$ of a polynomial on $\mathbb{R}^{n}$ which is harmonic and homogeneous of degree $l$. We write $\mathrm{SH}_{l}\left(\mathbb{S}^{n-1}\right)$ for the vector space of spherical harmonics of degree $l$ and $d_{l}$ its dimension. Two spherical harmonics of different degrees are orthogonal with respect to the usual scalar product on $L^{2}\left(\mathbb{S}^{n-1}, d \sigma\right)$.

Let $\left(E_{1}^{l}, \ldots, E_{d_{l}}^{l}\right)$ be an orthonormal basis of $S H_{l}\left(\mathbb{S}^{n-1}\right)$. For all $\zeta, \eta \in \mathbb{S}^{n-1}$, we put

$$
Z_{l}(\zeta, \eta)=\sum_{j=1}^{d_{l}} E_{j}^{l}(\zeta) \overline{E_{j}^{l}(\eta)}
$$

The function $\eta \mapsto Z_{l}(\zeta, \eta)$ is the zonal with pole $\zeta$ of degree $l$ and is independent of the choice of the $E_{j}^{l}$. Given $f \in L^{1}\left(\mathbb{S}^{n-1}, d \sigma\right)$, we define $\Pi_{l} f$ by

$$
\Pi_{l} f(\zeta)=\int_{\mathbb{S}^{n-1}} Z_{l}(\zeta, \eta) f(\eta) d \sigma(\eta)
$$

for all $\zeta \in \mathbb{S}^{n-1} ; \Pi_{l} f$ is a spherical harmonic of degree $l$ and, in the case $f$ is in $L^{2}\left(\mathbb{S}^{n-1}, d \sigma\right)$, it is the orthogonal projection of $f$ on $S H_{l}\left(\mathbb{S}^{n-1}\right)$. The series

$$
\sum_{l=0}^{+\infty} \Pi_{l} f
$$

is called the Fourier-Laplace series of $f$ (see [2] for more on spherical harmonics).

It is a classical result [7, p. 45] that the Fourier-Laplace series of an integrable function $f$ on $\mathbb{S}^{n-1}$ is $(C, \delta)$-summable in mean to $f$ when $\delta>(n-2) / 2$.

Given $\delta \geq 0$, a series $\sum_{m \geq 0} b_{m}$ of complex numbers is $(C, \delta)$-summable to $B \in \mathbb{C}$ if

$$
\lim _{m \rightarrow+\infty} \sum_{l=0}^{m}\left(\begin{array}{c}
m-l+\delta \\
\delta
\end{array}\right)\left(\begin{array}{c}
m+\delta \\
\delta
\end{array}\right)^{-1} b_{l}=B,
$$


where

$$
\left(\begin{array}{c}
k+\delta \\
\delta
\end{array}\right)=\frac{(\delta+1)(\delta+2) \cdots(\delta+k)}{k !} .
$$

In particular, a series is $(C, 0)$-summable to $B$ if and only if it converges to $B$. Moreover, if it is $(C, \delta)$-summable to $B$, it is $\left(C, \delta^{\prime}\right)$-summable to $B$ for all $\delta^{\prime} \geq \delta$ [3, pp. 96-100].

Finally, with the above notation we have, for any $\delta \geq 0$ and $d \in \mathbb{N}_{\geq 0}$,

$$
\lim _{m \rightarrow+\infty}\left(\begin{array}{c}
m-d+\delta \\
\delta
\end{array}\right)\left(\begin{array}{c}
m+\delta \\
\delta
\end{array}\right)^{-1}=1 .
$$

Indeed, this means that the series $\sum_{m \geq 0} b_{m}$, where $b_{d}=1$ and $b_{m}=0$ if $m \neq d$, is $(C, \delta)$ summable to 1 .

\section{Proof}

By the first remark in Section 2, we see that it will suffice to establish the proposition with $\Lambda$ a sphere of radius $r$ centred in $0: \Lambda=S(0, r)$.

We begin by assuming that $J_{d+(n-2) / 2}(2 \pi r) \neq 0$ for all $d \in \mathbb{N}_{\geq 0}$. We take a function $f$ integrable on $\mathbb{S}^{n-1}$ such that

$$
\int_{\mathbb{S}^{n-1}} e^{-2 \pi i x \cdot \eta} f(\eta) d \sigma(\eta)=0
$$

for all $x \in S(0, r)$. We must show that $f=0$ almost everywhere on $\mathbb{S}^{n-1}$. We choose $\delta>(n-2) / 2$ and calculate for $x \in S(0, r)$ :

$$
\begin{aligned}
\mid \sum_{l=0}^{m}( & \left.\left(\begin{array}{c}
m-l+\delta \\
\delta
\end{array}\right)\left(\begin{array}{c}
m+\delta \\
\delta
\end{array}\right)^{-1} \int_{\mathbb{S}^{n-1}} e^{-2 \pi i x \cdot \eta} \Pi_{l} f(\eta) d \sigma(\eta)\right) \mid \\
= & \mid \int_{\mathbb{S}^{n-1}} e^{-2 \pi i x \cdot \eta}\left(\sum_{l=0}^{m}\left(\begin{array}{c}
m-l+\delta \\
\delta
\end{array}\right)\left(\begin{array}{c}
m+\delta \\
\delta
\end{array}\right)^{-1} \Pi_{l} f(\eta)\right) d \sigma(\eta) \\
& -\int_{\mathbb{S}^{n-1}} e^{-2 \pi i x \cdot \eta} f(\eta) d \sigma(\eta) \mid \\
= & \left|\int_{\mathbb{S}^{n-1}} e^{-2 \pi i x \cdot \eta}\left(\sum_{l=0}^{m}\left(\begin{array}{c}
m-l+\delta \\
\delta
\end{array}\right)\left(\begin{array}{c}
m+\delta \\
\delta
\end{array}\right)^{-1} \Pi_{l} f(\eta)-f(\eta)\right) d \sigma(\eta)\right| \\
\leq & \int_{\mathbb{S}^{n-1}}\left|e^{-2 \pi i x \cdot \eta}\right| \cdot\left|\sum_{l=0}^{m}\left(\begin{array}{c}
m-l+\delta \\
\delta
\end{array}\right)\left(\begin{array}{c}
m+\delta \\
\delta
\end{array}\right)^{-1} \Pi_{l} f(\eta)-f(\eta)\right| d \sigma(\eta) \\
= & \int_{\mathbb{S}^{n-1}}\left|\sum_{l=0}^{m}\left(\begin{array}{c}
m-l+\delta \\
\delta
\end{array}\right)\left(\begin{array}{c}
m+\delta \\
\delta
\end{array}\right)^{-1} \Pi_{l} f(\eta)-f(\eta)\right| d \sigma(\eta)
\end{aligned}
$$

and this last integral tends to 0 for $m \rightarrow+\infty$ by our choice of $\delta$. Hence,

$$
\lim _{m \rightarrow+\infty} \sum_{l=0}^{m}\left(\begin{array}{c}
m-l+\delta \\
\delta
\end{array}\right)\left(\begin{array}{c}
m+\delta \\
\delta
\end{array}\right)^{-1} \int_{\mathbb{S}^{n-1}} e^{-2 \pi i x \cdot \eta} \Pi_{l} f(\eta) d \sigma(\eta)=0
$$


uniformly in $x \in S(0, r)$. Now, by [1, Lemma 9.10.2, p. 464], for any spherical harmonic $Y_{k}$ of degree $k$,

$$
\int_{\mathbb{S}^{n-1}} e^{-2 \pi i t \zeta \cdot \eta} Y_{k}(\eta) d \sigma(\eta)=2 \pi i^{k} t^{-(n-2) / 2} J_{k+(n-2) / 2}(2 \pi t) Y_{k}(\zeta),
$$

where $t \geq 0$ and $\zeta \in \mathbb{S}^{n-1}$. Writing $x=r \xi$ with $\xi \in \mathbb{S}^{n-1}$ in (3.1), we get

$$
\lim _{m \rightarrow+\infty} \sum_{l=0}^{m}\left(\begin{array}{c}
m-l+\delta \\
\delta
\end{array}\right)\left(\begin{array}{c}
m+\delta \\
\delta
\end{array}\right)^{-1} 2 \pi i^{l} r^{-(n-2) / 2} J_{l+(n-2) / 2}(2 \pi r) \Pi_{l} f(\xi)=0
$$

uniformly in $\xi \in \mathbb{S}^{n-1}$. Therefore, fixing $d \in \mathbb{N}_{\geq 0}$,

$$
\lim _{m \rightarrow+\infty} \int_{\mathbb{S}^{n-1}}\left(\sum_{l=0}^{m}\left(\begin{array}{c}
m-l+\delta \\
\delta
\end{array}\right)\left(\begin{array}{c}
m+\delta \\
\delta
\end{array}\right)^{-1} \frac{2 \pi i^{l} J_{l+(n-2) / 2}(2 \pi r)}{r^{(n-2) / 2}} \Pi_{l} f(\xi)\right) \overline{\Pi_{d} f(\xi)} d \sigma(\xi)=0
$$

or

$$
\lim _{m \rightarrow+\infty} \sum_{l=0}^{m}\left(\begin{array}{c}
m-l+\delta \\
\delta
\end{array}\right)\left(\begin{array}{c}
m+\delta \\
\delta
\end{array}\right)^{-1} \frac{2 \pi i^{l} J_{l+(n-2) / 2}(2 \pi r)}{r^{(n-2) / 2}}\left(\int_{\mathbb{S}^{n-1}} \Pi_{l} f(\xi) \overline{\Pi_{d} f(\xi)} d \sigma(\xi)\right)=0 .
$$

But

$$
\int_{\mathbb{S}^{n-1}} \Pi_{l} f(\xi) \overline{\Pi_{d} f(\xi)} d \sigma(\xi)=0
$$

if $l \neq d$. So, (3.2) reduces to

$$
\lim _{m \rightarrow+\infty}\left(\begin{array}{c}
m-d+\delta \\
\delta
\end{array}\right)\left(\begin{array}{c}
m+\delta \\
\delta
\end{array}\right)^{-1} 2 \pi i^{d} r^{-(n-2) / 2} J_{d+(n-2) / 2}(2 \pi r)\left\|\Pi_{d} f\right\|_{2}^{2}=0
$$

or, in view of the last remark in Section 2, to

$$
2 \pi i^{d} r^{-(n-2) / 2} J_{d+(n-2) / 2}(2 \pi r)\left\|\Pi_{d} f\right\|_{2}^{2}=0 .
$$

From our choice of $r$, we deduce that $\left\|\Pi_{d} f\right\|_{2}^{2}=0$, hence $\Pi_{d} f=0$, and this is true for any $d \in \mathbb{N}_{\geq 0}$. The Fourier-Laplace series of $f$ is therefore identically zero, so $(C, \delta)$ summable in mean to zero: $\|f\|_{1}=0$, that is, $f=0$ almost everywhere on $\mathbb{S}^{n-1}$.

Assume now that there exists $d \in \mathbb{N}_{\geq 0}$ such that $J_{d+(n-2) / 2}(2 \pi r)=0$. Choose as $f$ integrable on $\mathbb{S}^{n-1}$ a nonzero spherical harmonic of degree $d$. Then

$$
\int_{\mathbb{S}^{n-1}} e^{-2 \pi i x \cdot \eta} f(\eta) d \sigma(\eta)=2 \pi i^{d} r^{-(n-2) / 2} J_{d+(n-2) / 2}(2 \pi r) f(\xi),
$$

that is,

$$
\int_{\mathbb{S}^{n-1}} e^{-2 \pi i x \cdot \eta} f(\eta) d \sigma(\eta)=0
$$

for all $x=r \xi \in S(0, r)$. 
REMARK 3.1. The proposition is the exact generalization to $n \geq 2$ of the mentioned result of Lev and Sjölin, the supplementary $2 \pi$ in our statement coming solely from a different normalization of the Fourier transform.

REMARK 3.2. Equality (3.3) shows that if $\Lambda$ is the zero set of a homogeneous harmonic polynomial on $\mathbb{R}^{n}$, then $\left(\mathbb{S}^{n-1}, \Lambda\right)$ is not a Heisenberg uniqueness pair. This is the case, for example, in $\mathbb{R}^{n}$ with the union of the hyperplanes $x_{1}=0, x_{2}=0, \ldots, x_{n}=0$, and in $\mathbb{R}^{3}$ with the cone

$$
\frac{x^{2}}{a^{2}}+\frac{y^{2}}{b^{2}}=\frac{z^{2}}{a^{2}}+\frac{z^{2}}{b^{2}} .
$$

\section{References}

[1] G. E. Andrews, R. Askey and R. Roy, Special Functions (Cambridge University Press, Cambridge, 1999).

[2] H. Groemer, Geometric Applications of Fourier Series and Spherical Harmonics (Cambridge University Press, Cambridge, 1996).

[3] G. H. Hardy, Divergent Series (Clarendon Press, Oxford, 1949).

[4] H. Hedenmalm and A. Montes-Rodríguez, 'Heisenberg uniqueness pairs and the Klein-Gordon equation', Ann. of Math. (2) 173 (2011), 1507-1527.

[5] N. Lev, 'Uniqueness theorems of Fourier transforms', Bull. Sci. Math. 135 (2011), 135-140.

[6] P. Sjölin, 'Heisenberg uniqueness pairs and a theorem of Beurling and Malliavin', Bull. Sci. Math. 135 (2011), 125-133.

[7] C. D. Sogge, 'Oscillatory integrals and spherical harmonics', Duke Math. J. 53 (1986), 43-65.

FRANCISCO JAVIER GONZÁLEZ VIELI, Avenue de Montoie 45,

1007 Lausanne, Switzerland

e-mail: francisco-javier.gonzalez@gmx.ch 